\title{
Measurements and Modeling of Kinetic Inductance Microstrip Delay Lines
}

\author{
JEFFREY M. POND, MEMBER, IEEE, JOHN H. CLAASSEN, AND WILLIAM L. CARTER
}

\begin{abstract}
Superconducting microstrip, with phase velocities of about $0.01 c$, employing kinetic inductance have been fabricated using niobium nitride and a silicon dielectric. Delay lines using this phenomenon have several advantages for analog signal processing, including low loss and very compact size. Measurements of kinetic inductance microstrip delay lines were made in the frequency domain and the time domain. The results were compared to theoretical predictions and an accurate circuit model was developed. The circuit model was used to determine the frequency- and temperature-dependent losses of the delay line.
\end{abstract}

\section{INTRODUCTION}

$\mathrm{R}$ ECENT DEVELOPMENTS in high-transition-temperature superconductors [1], operating at liquid nitrogen temperatures, promise to revolutionize many areas of electronics. Even at liquid helium temperatures there has been work involving the use of superconducting microstrip and stripline to accomplish delay and other signal processing applications [2], [3]. By using microstrip or stripline geometry with the conductors replaced by superconductors, performance surpassing conventional techniques has been obtained.

It has been reported [4] that advantages, including compact size and velocity tunability, can result by exploiting kinetic inductance $\left(L_{k}\right)$, which is due to the inertial mass of the current carriers in a conductor. The difficulty in observing $L_{k}$ in a normal wire is evident by considering that the resistance per unit length of a conductor of cross-sectional area $A$ is

$$
R=m /\left(n e^{2}\right)(1 / A)(1 / \tau) .
$$

At frequency $\omega$, the kinetic reactance per unit length, in simplest form, is

$$
\omega L_{k}=m /\left(n e^{2}\right)(1 / A) \omega
$$

where $m, n, e$, and $\tau$ are the mass, density, charge, and collision time of an electron. Only if the conductor is superconducting is $\omega>1 / \tau$ for microwave frequencies, and hence the kinetic reactance can dominate the resistance.

The slowing of an electromagnetic wave on a superconducting transmission line due to the contribution of $L_{k}$ was pointed out by Pippard in 1947 [5]. The measurement of this slowing, using thin films, has been exploited as a

Manuscript received April 6, 1987; revised August 24, 1987. This work was supported in part by the Office of Naval Research.

The authors are with the Naval Research Laboratory, Washington, DC 20375.

IEEE Log Number 8717396. method of determining the penetration depth of thin superconducting films [6]. It is possible to construct a microstrip line where the dominant inductance is due to $L_{k}$ rather than the magnetic inductance, $L_{m}$. This occurs when more of the magnetic energy is stored in the superconductor than in the dielectric. Thus the dielectric layer must be thin compared to the penetration depth of the magnetic field into the superconductor. Solutions for electromagnetic propagation in a trilayer consisting of a lossy dielectric between two superconducting films have been presented for both the simple two-fluid model [7] and the Mattis-Bardeen theory for complex conductivity [8].

In addition, this technology has the unique capability of continuously varying the phase velocity by changing the temperature. This behavior is a result of the temperature dependence of the penetration depth of the magnetic field into a superconductor. This concept is applicable to microstrip phase shifters and tunable filters as well as variable delay lines.

\section{Numerical Calculations}

In attempting to exploit $L_{k}$ for microwave delay lines, the circumstances which give rise to the slowest possible phase velocity with minimum loss and dispersion are of interest. The development to follow uses the solution presented in [7] which uses the London theory and the two-fluid model. It is assumed that the dielectric thickness $(d)$ and the penetration depth $(\lambda)$ are small compared to the width $(w)$ of the microstrip. If $w$ is small compared to both the wavelength and the length of the line, then the phase velocity is

$$
\begin{aligned}
v_{p}=c\left[\epsilon _ { r } \left(1+\left(\lambda_{1} / d\right)\right.\right. & \operatorname{coth}\left(t_{1} / \lambda_{1}\right) \\
& \left.\left.+\left(\lambda_{2} / d\right) \operatorname{coth}\left(t_{2} / \lambda_{2}\right)\right)\right]^{-1 / 2}
\end{aligned}
$$

where $c$ is the speed of light in vacuum, $\epsilon_{r}$ is the relative permittivity, $t$ is the superconductor thickness, and the subscripts 1 and 2 refer to the strip and ground plane, respectively. In order to minimize the phase velocity, it is desirable to have $d \ll \lambda_{1}, d \ll \lambda_{2}, t_{1} \ll \lambda_{1}$, and $t_{2} \ll \lambda_{2}$. Expressions for the group velocity and the frequencydependent losses of the superconductors exist [7], [8]. Except for frequencies near the gap frequency, it has been shown that the differences between the two-fluid and the Mattis-Bardeen models are minimal [9]. The dielectric loss of the microstrip has been found to be the dominant loss and for these reasons the simpler two-fluid model is used 


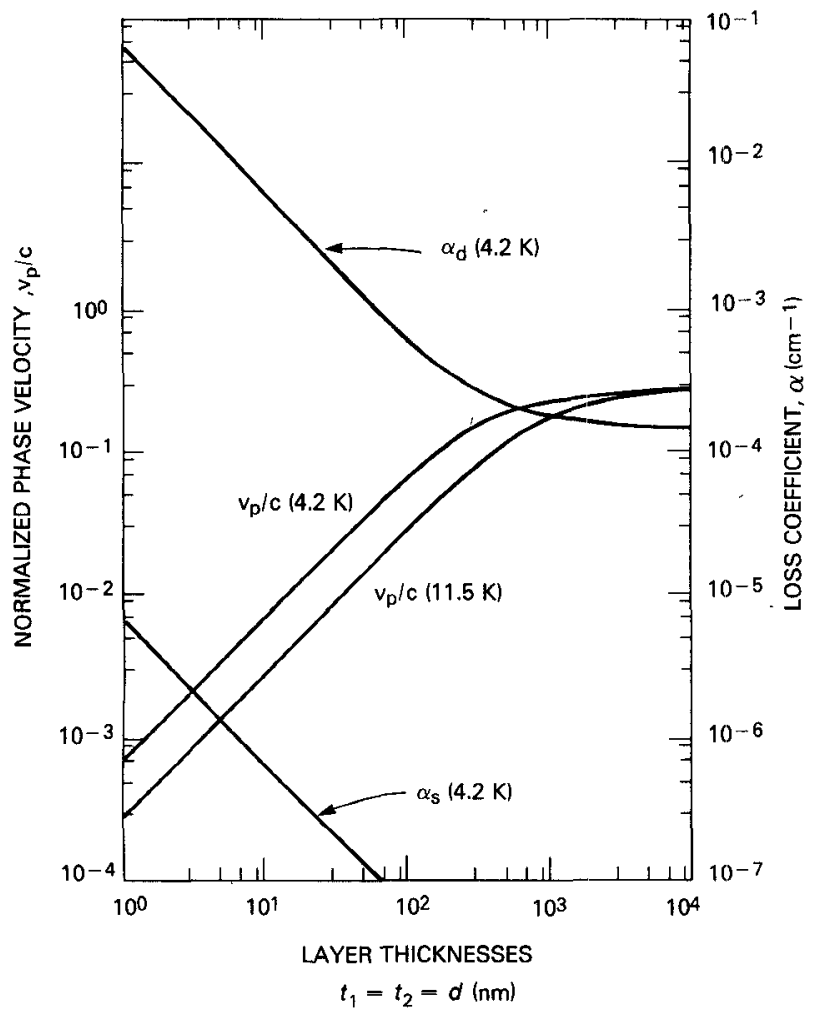

Fig. 1. $v_{p} / c$ at $4.2 \mathrm{~K}$ and $11.5 \mathrm{~K}, \alpha_{d}$ at $4.2 \mathrm{~K}$. and $\alpha_{s}$ at $4.2 \mathrm{~K}$ are plotted as a function of layer thickness for a $\mathrm{NbN} / \mathrm{Si} / \mathrm{NbN}$ delay line where $d=t_{1}=t_{2}$. The calculation uses $\epsilon_{t}=12, \mathrm{t}^{\prime \prime} / \epsilon^{\prime}=0.0002, T_{c}=12$ $\mathrm{K}, \sigma=10^{4} \mathrm{mhos} / \mathrm{cm}$, and $\lambda_{0}=3000 \AA$ at a frequency of $2.0 \mathrm{GHz}$.

for the following calculations. This model assumes that the temperature dependence of $\lambda$ is

$$
\lambda=\lambda_{0}\left[1-\left(T / T_{c}\right)^{4}\right]^{-1 / 2}
$$

where $T_{c}$ is the critical temperature and $\lambda_{0}$ is the penetration depth at $0.0 \mathrm{~K}$.

Fig. 1 shows the calculated dependence of the phase velocity of a $\mathrm{NbN} / \mathrm{Si} / \mathrm{NbN}$ microstrip as a function of the layer thickness of the microstrip at $4.2 \mathrm{~K}$ and $11.5 \mathrm{~K}$ where $T_{c}$ is $12 \mathrm{~K}$ for both layers. Film thicknesses of $100 \AA$ result in a substantial reduction in the phase velocity. These curves assume that all three layers are of equal thickness and that the penetration depth and conductivity are independent of film thickness. For layers which are thin compared to the penetration depth, a majority of the magnetic energy is stored in the superconductors. The real parts of the complex propagation coefficient due to dielectric loss $\left(\alpha_{d}\right)$ and superconductor loss $\left(\alpha_{s}\right)$ at $4.2 \mathrm{~K}$ were calculated and are also shown. The superconductor losses were calculated using [6], while the dielectric losses were determined using

$$
\alpha_{d}=\left(\omega / 2 v_{p}\right)\left(\epsilon^{\prime \prime} / \epsilon^{\prime}\right)
$$

where $\epsilon^{\prime}$ and $\epsilon^{\prime \prime}$ are the real and imaginary parts of the permittivity, and would dominate the superconductor losses at $2.0 \mathrm{GHz}$ for $\epsilon^{\prime \prime} / \epsilon^{\prime}>10^{-7}$. Deposited films usually have substantially higher values of $\epsilon^{\prime \prime} / \epsilon^{\prime}$, and a value of $2 \times 10^{-4}$ has been assumed for the calculation shown in Fig. 1.
The velocity given by (3) can be compared with the expression in terms of capacitance per unit length $(C)$ and inductance per unit length $(L)$ where

$$
v_{p}=(L C)^{-1 / 2} \text {. }
$$

Because $d \ll w, C$ is given by the parallel-plate expression

$$
C=\epsilon_{0} \epsilon_{r} w / d .
$$

It follows that $L$ is given by

$$
\begin{aligned}
& L=\mu_{0} d\left[1+\left(\lambda_{1} / d\right) \operatorname{coth}(\right.\left.t_{1} / \lambda_{1}\right) \\
&\left.+\left(\lambda_{2} / d\right) \operatorname{coth}\left(t_{2} / \lambda_{2}\right)\right] / w
\end{aligned}
$$

where $\epsilon_{0}$ and $\mu_{0}$ are the permittivity and permeability of free space. The first term of (8) is due to $L_{m}$ and the second and third terms are due to $L_{k}$ of the superconducting layers. Further simplifications result for certain situations of practical interest. Assuming $t_{1}=t_{2}=t$ and $\lambda_{1}=\lambda_{2}=\lambda$, then if $L_{k} \gg L_{m}$ and $t \ll \lambda, v_{p}$ and $Z_{c}$ reduce to

$$
Z_{c}=\lambda / w\left[\frac{2 d \mu_{0}}{\epsilon_{0} \epsilon_{r} t}\right]^{1 / 2}
$$

and

$$
v_{p}=1 / \lambda\left[\frac{d t}{2 \mu_{0} \epsilon_{0} \epsilon_{r}}\right]^{1 / 2} .
$$

From these equations it is possible to construct a wide variety of curves for practical situations which show the functional dependence of the velocity and impedance of a microstrip line as a function of the geometry. Since $w \gg d$, the use of equations developed from the parallel-plate analysis is valid.

The dependencies of $v_{p}$, normalized to $c$ and $c / \sqrt{\epsilon_{r}}$, and $Z_{c}$ as a function of $t$ for several values of $w$ (in $\mu \mathrm{m}$ ) are plotted in Fig. 2. It is assumed that $d=1000 \AA, \lambda=3000$ $\AA$, and $\epsilon_{r}=12$. The slowing factor due to $L_{k}$ varies from less than 0.04 to greater than 0.20 as $t$ is decreased. For $t$ in the range of about $100 \AA$ to $200 \AA$, very practical values for $Z_{c}$ can be obtained using high-quality optical lithography. This fact is relevant to the design of tunable filters where the electrical length of resonant elements can be varied with temperature, since $\lambda$ is a strong function of temperature near $T_{c}$, but may also be accomplished optically and possibly electronically by the generation of quasi-particles. An examination of (9) and (10) reveals that lower $v_{p}$ and higher $Z_{c}$ can be simultaneously realized by increasing $\lambda$, either through a larger value of $\lambda_{0}$ of the film or through operation of the microstrip at a temperature closer to $T_{c}$. These calculations have assumed that $\lambda_{0}$ is a constant. In practice $\lambda_{0}$ and $T_{c}$ are often dependent on the deposition parameters and thickness of a sputtered film. Thus, rather than being design curves for kinetic inductance microstrip lines, Fig. 2 is representative of the geometries required to obtain specific characteristics. 


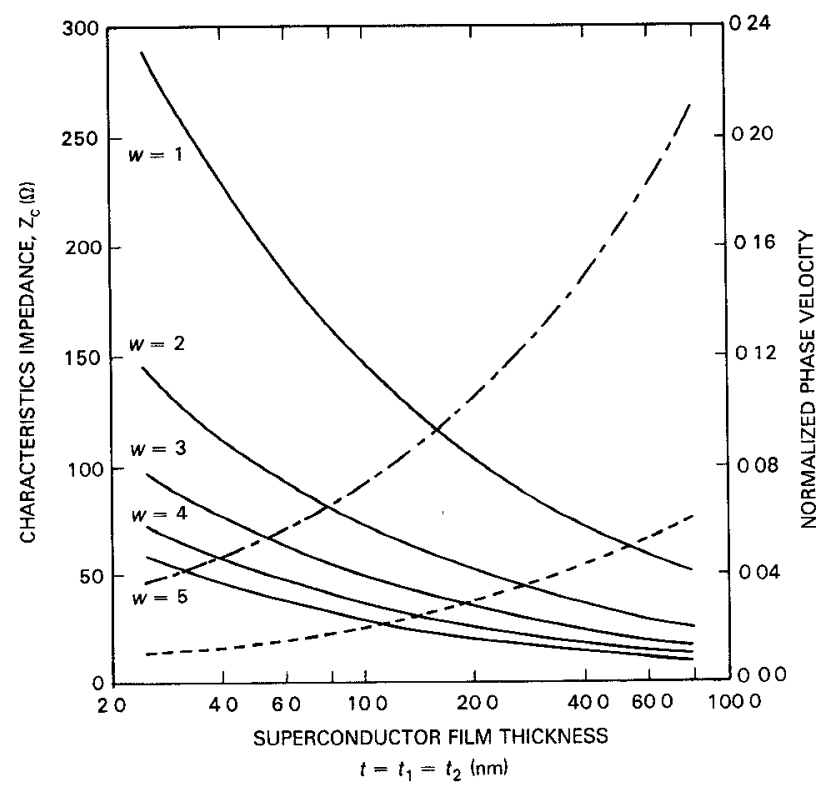

Fig. $2 \quad Z_{r}(-), v_{p} / c(---)$ and $\sqrt{\epsilon_{r}} v_{p} / c(--)$ plotted versus $t$ for several $w(1 \mathrm{n} \mu \mathrm{m})$ with $d=1000 \AA, \lambda=3000 \AA$, and $\epsilon_{r}=12.0$.

\section{Device Fabrication and Test Fixture}

The devices were fabricated in an ultra-high-vacuum $\mathrm{RF}$ sputtering system. A $150-\AA \mathrm{NbN}$ ground plane was deposited on a quartz substrate followed by $250 \AA$ of hydrogenated silicon $(\mathrm{Si}: \mathrm{H})$. These layers were patterned and plasma etched to open holes in the ground plane for the microstrip contact pads. Then, $150 \AA$ of $\mathrm{Si}: \mathrm{H}$ was deposited followed by a second $\mathrm{NbN}$ layer of $140 \AA$. A description of the thin film deposition system and complete parameters of the depositions can be found in [10] and [4], respectively.

Contact pads were then patterned which were connected by a $25-\mu \mathrm{m}$-wide, $20.1-\mathrm{cm}$-long microstrip. The contact pads were then covered by $100 \AA$ of $\mathrm{Cr}$ and $1000 \AA$ of $\mathrm{Au}$. It was found that in order to have a repeatable contact it was necessary to melt some indium on the Au contacts. The sample was mounted in a fixture which used 0.141 -inch semirigid coaxial cable with a spring-loaded center conductor to contact the contact pads perpendicular to the substrate surface. The test fixture was then mounted in a cryostat with $110-\mathrm{cm}$ coaxial cable feedthroughs to room temperature.

\section{EXPERIMENTAL ResUlts}

Frequency responses were measured using Hewlett Packard 8409 and 8510 automatic network analyzers. The power incident on the input coaxial cable feedthrough was set at $100 \mu \mathrm{W}$ since it is possible to overdrive the microstrip line. A simple calculation, assuming a $4-\Omega$ line at resonance, gives an electric field in the dielectric of $5000 \mathrm{~V} / \mathrm{cm}$. The scattering matrix of the delay line and the feedthrough cables was measured in $50-\mathrm{MHz}$ and $100-\mathrm{MHz}$ frequency bands around $0.1 \mathrm{GHz}, 0.5 \mathrm{GHz}, 1.0 \mathrm{GHz}$, and $2.0 \mathrm{GHz}$ with a $1.0-\mathrm{MHz}$ sample increment. These measurements were made at $4.2 \mathrm{~K}, 7.3 \mathrm{~K}$, and $9.0 \mathrm{~K}$.

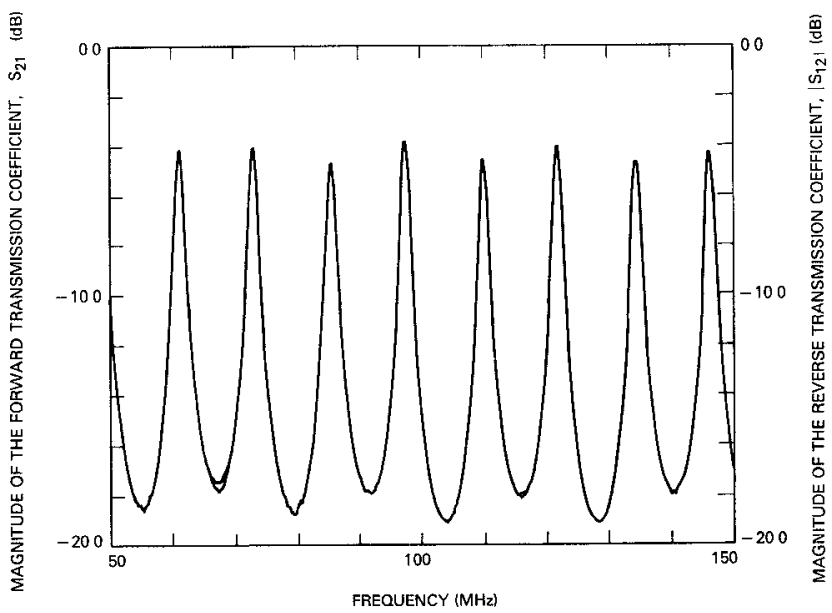

Fig 3. Magnitude of the transmission response of the delay line at 4.2 $\mathrm{K}$ over the frequency range from $50 \mathrm{MHz}$ to $150 \mathrm{MHz}$.

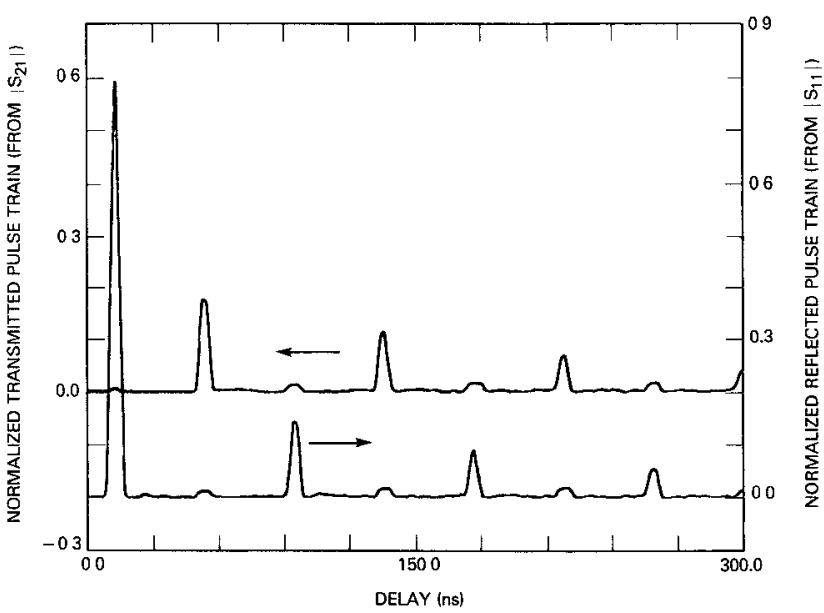

Fig. 4. Reflected and transmitted time-domain response of the delay line obtained from Fourner-transformed frequency-domain data.

Fig. 3 shows representative data for the magnitude of the transmission coefficients for the delay line and test fixture at $4.2 \mathrm{~K}$. The delay line is reciprocal and has a very repeatable resonance signature, indicating relatively low loss within the microstrip. The sharp peaks of $\left|S_{21}\right|$ correspond to frequencies where the length of delay line is an integral number of half-wavelengths. This is due to the large impedance mismatch and discontinuity, which causes the delay line to appear as a coupled cavity. Thus

$$
v_{p}=2 l \Delta f
$$

where $l$ is the length of the line and $\Delta f$ is the separation between peaks in $\left|S_{21}\right|$. The value of $\Delta f$ is approximately $12 \mathrm{MHz}$, which translates to a phase velocity of $0.016 c$. Similar measurements were obtained at other frequencies and temperatures, and the resonant peaks remained well defined up to at least $2.0 \mathrm{GHz}$, which corresponds to approximately 80 wavelengths in the delay line.

The time-domain response of the line is shown in Fig. 4. These data were obtained using the time-domain function of the HP 8510 and a frequency band of $0.05 \mathrm{GHz}$ to 0.50 $\mathrm{GHz}$. These measurements agree with transient measurements made using a pulse generator [4]. Due to the large 
mismatch, most of the incident pulse is reflected. The pulse in the microstrip undergoes multiple reflections, hence the series of equally spaced pulses of decreasing amplitude. The time-domain and frequency-domain data were used to accurately determine the phase velocity of the delay line as a function of temperature. Extraction of the losses and their behavior was more difficult. Discussion of these topics follows the development of an equivalent circuit model.

\section{Circuit Modeling}

Computer aided design and analysis techniques were applied to this structure not only to determine some of the embedded parameters of the microstrip line but also to demonstrate the ease of designing these structures. Modeling of this microwave circuit was done using EEsof's Touchstone program. The microstrip transmission line was simulated as a floating physical transmission line with the same length as the actual microstrip. The very slow phase velocity was simulated by choosing an appropriately high value for the effective relative dielectric constant $\left(\epsilon_{e}\right)$ of the transmission line. The input and output coax cables were modeled as transmission lines with an experimentally determined loss.

An examination of Fig. 3 indicates an interesting signature in the frequency response. The minima of the response have a periodicity in amplitude of twice the frequency period of the resonances (the amplitude variations in the maxima are due to the discretization of the frequency measurement). This correlates to the timedomain response of Fig. 4, where small pulses can be seen halfway between the major pulses of both pulse trains. This behavior was attributed to a short parallel transmission path between the input and output ports of the delay line. Two paths exist; one is a crosstalk between the center conductors of the input and output coax at the transition to the microstrip, which was modeled by the parallel combination of a capacitor and a lossy inductor. Due to the near field of the cryogenic environment, these parameters were determined by best fit to the data. The second parallel path is through the substrate, since the "ground plane" of the microstrip was not actually at ground potential but was connected to ground through the superconducting film which has an associated $L_{k}$. This second path was modeled by a short capacitively coupled transmission line between the input and output port contact pads of the delay line. Fig. 5 shows the forward and reverse transmission pulses due to the parallel conduction paths for a control device in which the delay line was not connected.

The equivalent circuit is shown in Fig. 6 . The delay line was modeled by a floating transmission line $20.1 \mathrm{~cm}$ long. The $\epsilon_{e}$ and $Z_{c}$ of the transmission line were allowed to vary with temperature to account for the temperature dependence of the penetration depth. The line loss was modeled as a shunt conductance for each frequency band, but was allowed to vary with temperature and the frequency band in order to achieve a best fit. A resistor, $R_{c}$, at each end of the delay line modeled the contact loss to the input

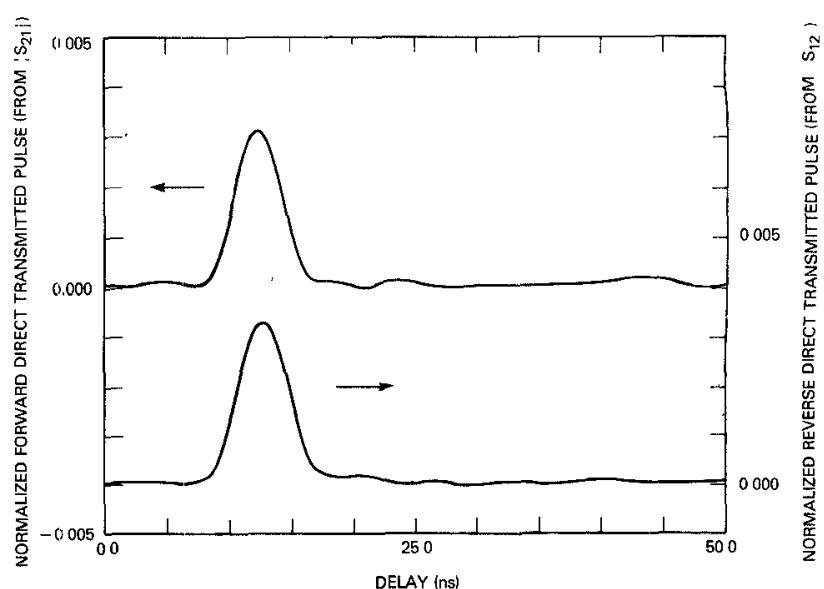

Fig 5. Direct transmitted pulses due to the parallel transmission paths which were measured using a control device

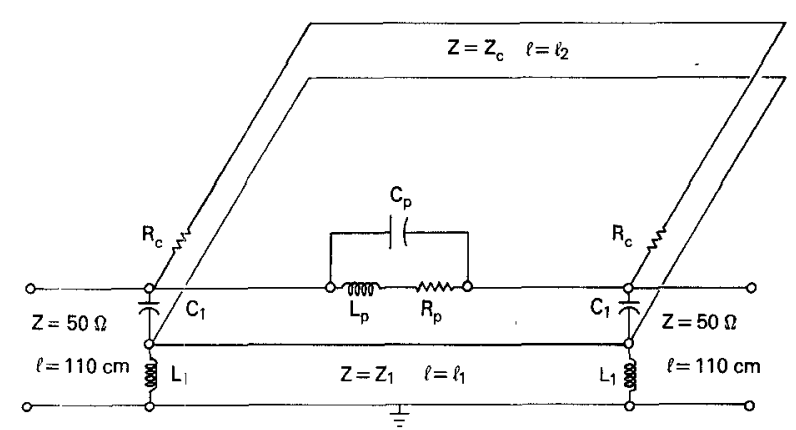

Fig 6. Equivalent circuit which accurately models the device response.

and output coax. The other conductor of the delay line was connected to ground through inductors $\left(L_{1}\right)$ which account for the $L_{k}$ of the lower superconductor between the device region and ground. The transmission line representing the short path through the substrate was capacitively coupled $\left(C_{1}\right)$ to the input and output transmission lines. The direct crosstalk path between the center conductors of the input and output coax cables was modeled by $C_{p}, L_{p}$, and $R_{p}$. The results of the circuit modeling, the element values used, and the physical parameters extracted are presented in the following section.

\section{Discussion}

Time-domain and frequency-domain measurements were used to accurately measure the phase velocity of the delay line. For the delay line under consideration, it was assumed that both superconducting films were $145 \AA$ thick. It was further assumed that both films had the same $T_{c}$ and $\lambda_{0}$. Thus (9) and (10) were used to analyze the measured results and the data extracted using the equivalent circuit model. The measured phase velocity, normalized to the speed of light in vacuum, is shown in Fig. 7 as a function of temperature. The circles represent the measured values while the solid line is the predicted response using (4) and (10). The transition temperature $12.15 \mathrm{~K}$, was determined by observing the temperature at which no signal was transmitted. It should be noted that there was very good agreement between the measured velocity and 


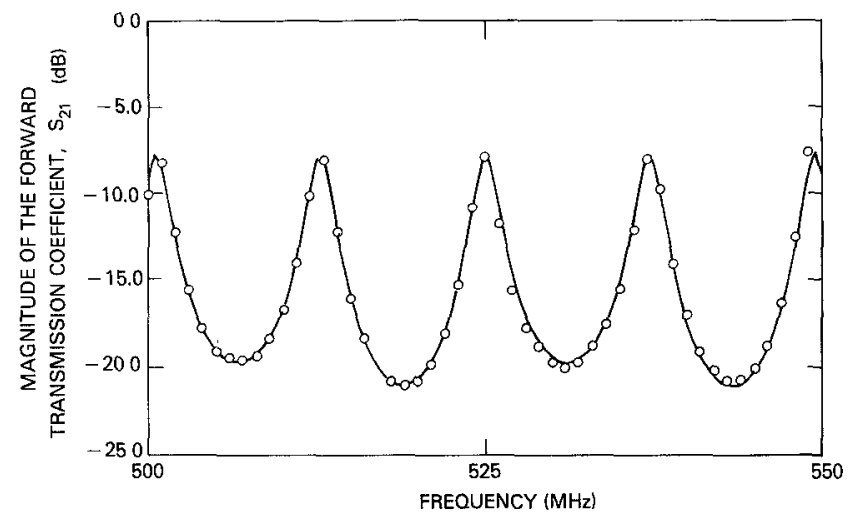

Fig. 7. Measured $\eta_{p}\left(0^{\circ}\right.$ plotted as a function of temperature and a comparison to the expected behavior ( - ) from (4).

the predicted response using (4). These results were used to calculate the $0.0 \mathrm{~K}$ phase velocity, $v_{p 0}=4.94 \times 10^{8} \mathrm{~cm} / \mathrm{s}$.

An earlier analysis [4] used a simple graphical technique to determine the loss of the delay line as a function of frequency and temperature. The results of this analysis were that the loss of the delay line was linear with frequency, as would be expected from a dielectric loss tangent. However, the loss when projected to zero frequency was not zero as would be expected with a dielectric loss tangent. Furthermore the loss was very dependent on temperature. That analysis assumed that the transitions were lossless, which if incorrect may account for the prediction of loss at zero frequency.

The important temperature-dependent parameters from the circuit modeling are $Z_{c}, \epsilon_{e}$, and the shunt conductance, $\sigma_{s}$, of the delay line. It was found that the circuit model discussed in the previous section could quite accurately reproduce the measured frequency response, as is shown in Fig. 8. The circles are the measured response at $4.23 \mathrm{~K}$ from the network analyzer, and the solid line is the frequency response using the circuit model. Similar agreement was found at $7.28 \mathrm{~K}$ and $9.00 \mathrm{~K}$. For this frequency band the values of the temperature-independent parameters used to achieve these three fits were $C_{p}=5 \mathrm{pF}$, $L_{p}=20 \mathrm{nH}, C_{1}=1 \mathrm{pF}, R_{c}=5 \Omega$, and $Z_{1}=50 \Omega$. The model is relatively sensitive to all these values except $C_{1}$. The values of the temperature dependent parameters are shown in Table I.

The temperature dependence of $\epsilon_{e}$ and $Z_{c}$ exhibited the correct functional behavior. Since the results shown in Fig. 7 confirmed the validity of (4), and since $\epsilon_{e}$ as used in the circuit modeling relates directly to $v_{p}$ as

$$
v_{p} / c=\left(\epsilon_{e}\right)^{-1 / 2}
$$

then it follows from (4) and (10) that

$$
v_{p 0} / v_{p}=\lambda / \lambda_{0}=\left(\epsilon_{e} / \epsilon_{e 0}\right)^{1 / 2}
$$

where $v_{p 0}$ and $\epsilon_{e 0}$ are $v_{p}$ and $\epsilon_{e}$ at $0.0 \mathrm{~K}$. Similarly, using (4) and (9),

$$
Z_{c 0} / Z_{c}=\lambda_{0} / \lambda
$$

where $Z_{c 0}$ is $Z_{c}$ at $0.0 \mathrm{~K}$. Equations (13) and (14) can be

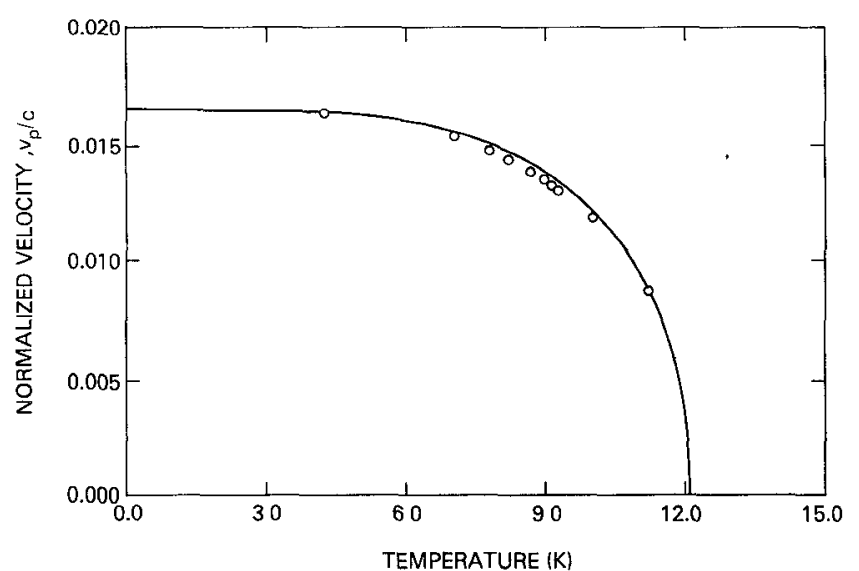

Fig 8. The equivalent circuit response (- - and measured response ( $)$ are compared for the $500 \mathrm{MHz}$ to $550 \mathrm{MHz}$ bandwidth at $4.23 \mathrm{~K}$.

TABLE I

\begin{tabular}{|c|c|c|c|}
\hline & $4.23 \mathrm{~K}$ & $7.28 \mathrm{~K}$ & $9.00 \mathrm{~K}$ \\
\hline$\epsilon_{u}$ & $\overline{3725}$ & $\overline{4320}$ & 5485 \\
\hline$Z(\Omega)$ & 3.5 & 3.75 & 4.2 \\
\hline$\sigma,($ siemens $/ \mathrm{cm})$ & 0.0018 & 0.0032 & 0.0045 \\
\hline$L_{1}(\mathrm{nH})$ & 0.4 & 0.425 & 0.48 \\
\hline$R_{p}(\Omega)$ & 0.0 & 3.0 & 10.0 \\
\hline
\end{tabular}

Thmptrature-Dependent Circuit Parameters Used for the $500 \mathrm{MHz}$ TO $550 \mathrm{MHz}$ BAND

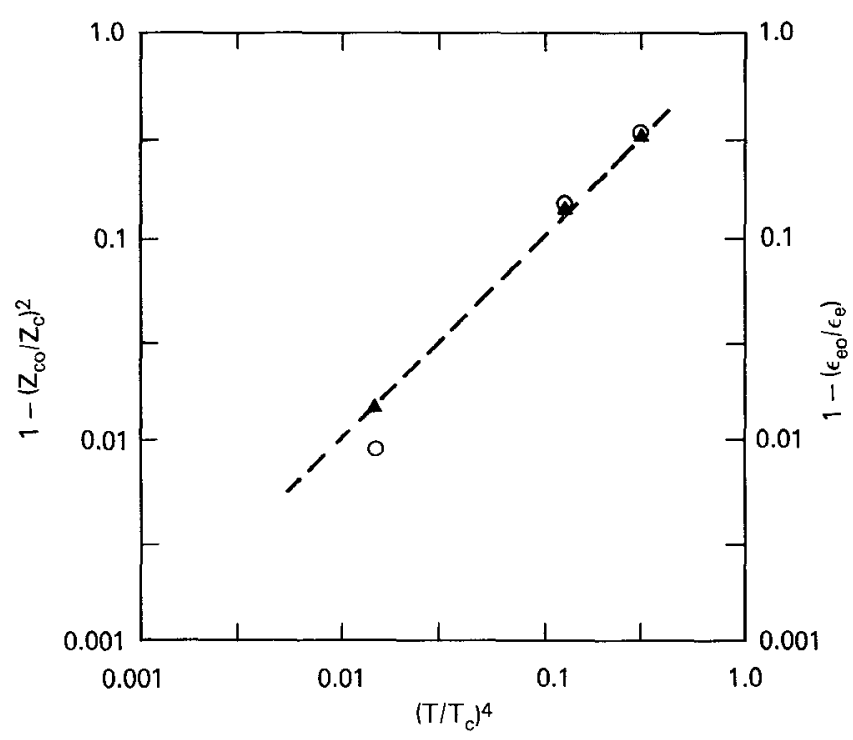

Fig 9 The temperature dependence of $Z_{c}(0)$ and $\epsilon_{e}(\boldsymbol{\Lambda})$ are compared to their expected functional dependence (---) from (4), (9), and (10)

rewritten using (4) as

$$
1-\epsilon_{e 0} / \epsilon_{e}=\left(T / T_{c}\right)^{4}
$$

and

$$
1-\left(Z_{c 0} / Z_{c}\right)^{2}=\left(T / T_{c}\right)^{4} .
$$

The values found from the circuit model and listed in Table I are plotted in Fig. 9 and compared to the theoretical plots of (15) and (16). The agreement is quite good and justifies the accuracy of the circuit model. The relationships 


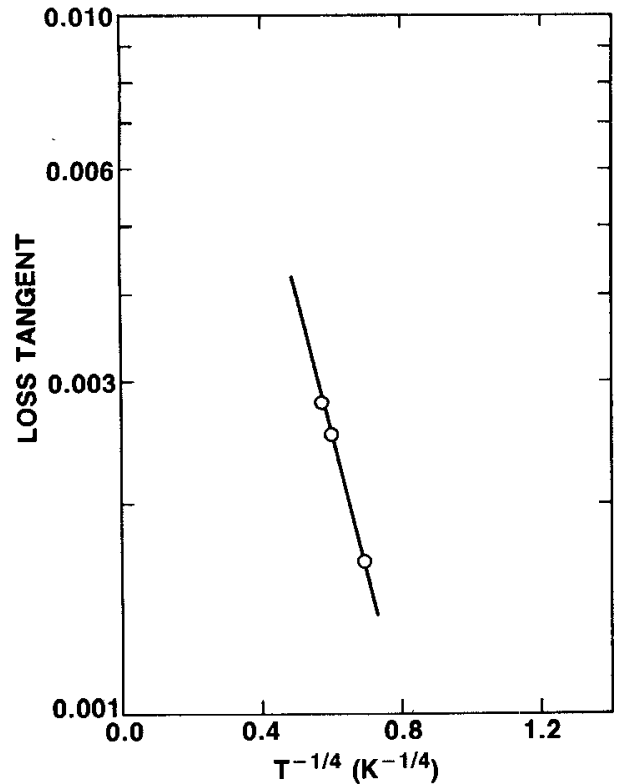

Fig 10. The loss tangent determined from the circtit model is plotted versus $T^{-1 / 4}$. The straight line is consistent with hopping conduction.

given by (15) and (16) were used in conjunction with the values of Table I to compute the values of $Z_{c 0}$ and $\epsilon_{e 0}$ to be $3.49 \Omega$ and 3691 , respectively. These values are consistent with $\lambda_{0}=3200 \AA$ and $\epsilon_{r}=10.5$, which are reasonable values for these films.

The temperature dependence of the loss as characterized by $\sigma_{s}$ is plotted against temperature to the negative onequarter power in semilog form as shown in Fig. 10. The behavior of this curve, although not over a large temperature range, was found to be a straight line. The vertical axis is not a plot of $\sigma_{s}$ but of the normalized parameter $\sigma_{s} /\left(\omega \epsilon_{e}\right)$, which can also be expressed as the dielectric loss tangent, $\epsilon^{\prime \prime} / \epsilon^{\prime}$, where $\epsilon^{\prime}$ and $\epsilon^{\prime \prime}$ are the real and imaginary parts, respectively, of a complex permittivity. This quantity relates to the real part of the complex propagation coefficient, $\alpha$, where

$$
\alpha=\omega\left(\mu_{0} \epsilon_{e}\right)^{1 / 2}\left[\sigma_{s} /\left(2 \omega \epsilon_{e}\right)\right] .
$$

A frequency value of $525 \mathrm{MHz}$ was used in these calculations since it was the center of the band. It was necessary to plot the normalized loss parameter rather than $\sigma_{s}$ since in the circuit model the physical length of the delay line was held constant while the electromagnetic length of the line was allowed to vary through the temperature dependence of $\epsilon_{e}$. The straight line behavior of Fig. 10 is consistent with a frequency-dependent hopping conduction model for the Si dielectric [11]. Comparison with the results of [4] using the graphical technique shows excellent agreement for the loss.

Two additional parameters of the equivalent circuit model, which were important for accurately matching the data, are $R_{p}$ and $R_{c}$. It was determined that to obtain good correlation with the data the value of $R_{p}$ must be temperature dependent. This is attributed to the temperature-dependent conductivity of the Si electric. Since most of the substrate surface is not covered by the top superconducting film, which was etched away to define the microstrip pattern, the dielectric surface acts as a lossy boundary to the crosstalk signal between the input and output coaxial cables. The pressure contact between the coax cable and the microstrip contact pad results in $R_{c}$.

\section{CONCLUSIONS}

A kinetic indúctance microwave delay line was developed and demonstrated in the $0.1 \mathrm{GHz}$ to $2.0 \mathrm{GHz}$ frequency range. The particular advantage of this technique is the possibility of obtaining very large variable delays in a very small area due to the reduced velocity and small coupling between lines. In addition these devices have the potential for high-frequency operation. These devices can be described and hence designed by common computer aided design systems. Due to the processing compatibility with Josephson tunnel junction technology, this technology allows the possibility of accomplishing sophisticated signal processing functions as well as combined analog and digital functions. The primary area for improvement of this device is the reduction of the loss in the dielectric film. The loss mechanisms of the film have been measured and identified with the aid of the circuit model. Much lower losses, at least an order of magnitude, should be obtainable with high-quality dielectric films. Other arreas of emphasis for future work will be in the implementation of other devices such as phase shifters, tunable filters, taps, and coupled lines. Accurate and repeatable control of $v_{p}$ is important for this technology.

\section{ACKNOWLEDGMENT}

The authors would like to acknowledge the helpful discussions of Dr. D. Webb, Dr. M. Nisenoff, and Dr. E. Cukauskas.

\section{REFERENCES}

[1] M. K. Wu, J. R Ashburn, C. J. Torng, P. H. Hor, R. L. Meng, L. Gao, Z. J. Huang, Y Q. Wang, and C. W. Chu, "Supérconductivity at $93 \mathrm{~K}$ in a new mixed-phase $\mathrm{Y}-\mathrm{Ba}-\mathrm{Cu}-\mathrm{O}$ compound system at ambient pressure," Phys. Rev. Lett., vol. 58, no. 9, pp. 908-910, 1987

[2] D. A. Gandolfo, A. Boornard, and L. C. Morris, "Superconductive microwave meander lines," J. Appl. Phys., vol. 39, pp. 2657-2660, May 1968.

[3] S. A. Reible, "Wideband analog signal processing with superconductive circuits," in 1982 IEEE Ultrasontcs Symp. Dlg., pp. 190-201.

[4] J. M. Pond, J. H. Claassen, and W. L. Carter, "Kinetic inductance microstrip delay lines," IEEE Trans. Magn., vol. MAG-23, no. 2, pp. 903-907, Mar. 1987.

[5] A. B. Pippard, "The surface impedance of superconductor and normal metals at high frequencies, III. The relationship between impedance and superconducting penetration depth," Proc. Roy' Soc Lond., vol. A191, pp. 399-415, 1947

[6] W H. Henkels and C. J. Kircher, "Penetration depth measurements on type II superconducting films," IEEE Trans Magn.. vol. MA.G-13, no. 1, Jan. 1977

[7] J. C. Swihart, "Field solution for a thin-film superconducting strip transmission line," J. Appl. Phys., vol. 32, pp. 461-469, Mar. 1961

[8] P. V Mason and R. W Gould, "Slow-wave structures utilizing superconducting thin film transmission lines," $J$ Appl. Phys., vol. 40, pp 2039-2051, Apr. 1969 
[9] R. L. Kautz, "Picosecond pulses on superconducting striplines," $J$. Appl. Phys., vol, 49, pp. 308-314, Jan. 1978.

[10] E. J. Cukauskas and W. L. Carter, "Niobium nitride based Josephson junctions with unoxidized silicon barriers," IEEE Trans. Magn., vol. MAG-23, no. 2, pp. 669-672, Mar. 1987.

[11] K. Secger, Semiconductor Physics. New York: Springer-Verlag, 1973, pp. 467.

W

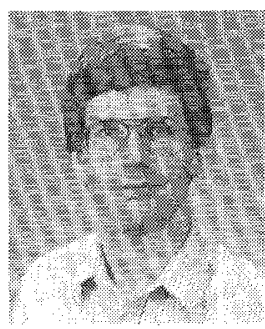

Jeffrey M. Pond (S'82-M'82) was born in South Haven, MI, in 1956. He received the B.S. degree in electrical engineering from Michigan State University in 1978 and the M.S. and Ph.D. degrees in electrical engineering from the University of Michigan in 1979 and 1982, respectively.

In 1982 he joined the Naval Research Laboratory in Washington, DC. 'His research interests have centered on semiconductor and superconductor devices for microwave ap-

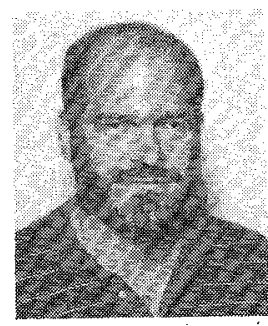

John H. Claassen was born in Norman, OK, on October 11, 1943. He received the B.S. degree from Wheaton College, Wheaton, IL, in 1965, and the Ph.D. degree from Cornell University, Ithaca, NY, in 1972. From 1972 to 1977 he was a research associate at the University of California, Berkeley. Since 1977 he has been employed as a Research Physicist at the Naval Research Laboratory, Washington, DC.

\section{幽}

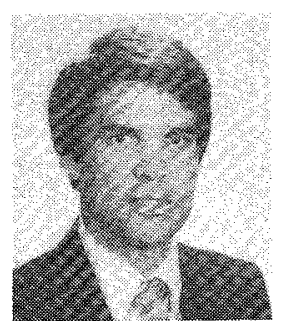

William L. Carter was born in Lynn, MA, on December 4, 1953. He received the B.A. degree from Carleton College in 1976 and the Ph.D. degree in physics from Stanford University in 1983.

In 1983 he joined the U.S. Naval Research Laboratory Electronics Technology Division, where he has worked on thin-film growth and thermal processing of advanced superconducting materials and devices for use in microwave and millimeter-wave components. 